\title{
SUMO modification is required for in vivo Hox gene regulation by the Caenorhabditis elegans Polycomb group protein SOP-2
}

Hong Zhang, Gromoslaw A Smolen, Rachel Palmer, Andrea Christoforou, Sander van den Heuvel \& Daniel A Haber

Post-translational modification of proteins by the ubiquitinlike molecule SUMO (sumoylation) regulates their subcellular localization and affects their functional properties in vitro ${ }^{1,2}$, but the physiological function of sumoylation in multicellular organisms is largely unknown. Here, we show that the $C$. elegans Polycomb group (PcG) protein SOP-2 interacts with the SUMO-conjugating enzyme UBC-9 through its evolutionarily conserved SAM domain. Sumoylation of SOP-2 is required for its localization to nuclear bodies in vivo and for its physiological repression of Hox genes. Global disruption of sumoylation phenocopies a sop-2 mutation by causing ectopic Hox gene expression and homeotic transformations. Chimeric constructs in which the SOP-2 SAM domain is replaced with that derived from fruit fly or mammalian PcG proteins, but not those in which the SOP-2 SAM domain is replaced with the SAM domains of non-PcG proteins, confer appropriate in vivo nuclear localization and Hox gene repression. These observations indicate that sumoylation of PcG proteins, modulated by their evolutionarily conserved SAM domain, is essential to their physiological repression of Hox genes.

\footnotetext{
Figure 1 Interaction of SOP-2 with UBC-9 and sumoylation. (a) Results of yeast two-hybrid screens using the $C$ terminus of SOP-2 as bait. The strength of the protein interaction is graded on the basis of comparison with controls: human Rb and E2F-1 interaction (+, faint blue color after $24 \mathrm{~h}$ in X-gal assay) and rat c-Fos and mouse c-Jun interaction (+++, blue color within $1 \mathrm{~h}$ in X-gal assay). (b) Interaction of the wild-type SOP-2 SAM domain with itself, with the SOP-2(bx91) mutant protein and with UBC-9. The bx91 mutation substantially reduces its interaction with UBC-9 but not with the wild-type SOP-2 SAM domain. (c) In vitro and in vivo sumoylation of SOP-2. In vitro sumoylation was carried out using labeled SOP-2 and purified sumoylation components. In vivo sumoylation of hemagglutinin-tagged SOP-2 and the SOP-2(bx91) mutant was done after transfection and expression in mammalian U2OS cells. Cell lysates were analyzed by immunoprecipitation (IP) with antibody to SUMO and western blotting with antibody to hemagglutinin.
}

a

\begin{tabular}{|c|cc|}
\multicolumn{3}{c}{ Summary of yeast two-hybrid experiments } \\
\hline SOP-2 interactors & $\begin{array}{c}\text { Independent } \\
\text { clones }(\mathrm{n})\end{array}$ & $\begin{array}{c}\text { Strength } \\
\text { of interaction }\end{array}$ \\
\hline SOP-2 & 23 & +++ \\
UBC-9 & 3 & ++ \\
\hline
\end{tabular}

b
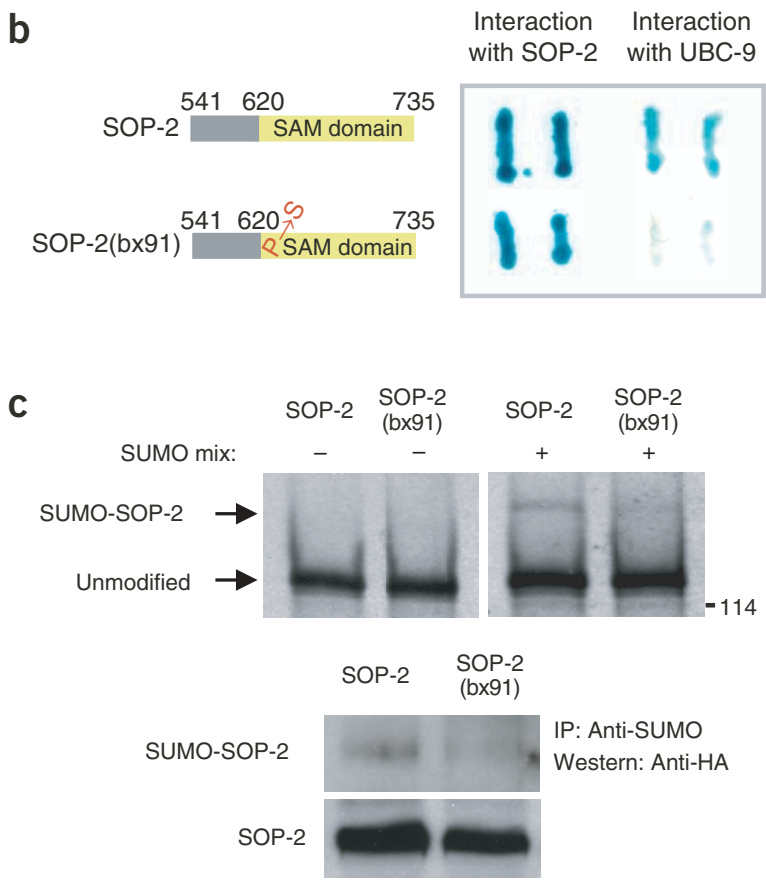

Massachusetts General Hospital Cancer Center and Harvard Medical School, Charlestown, Massachusetts 02129, USA. Correspondence should be addressed to D.A.H. (haber@helix.mgh.harvard.edu).

Published online 11 April 2004; doi:10.1038/ng1336 

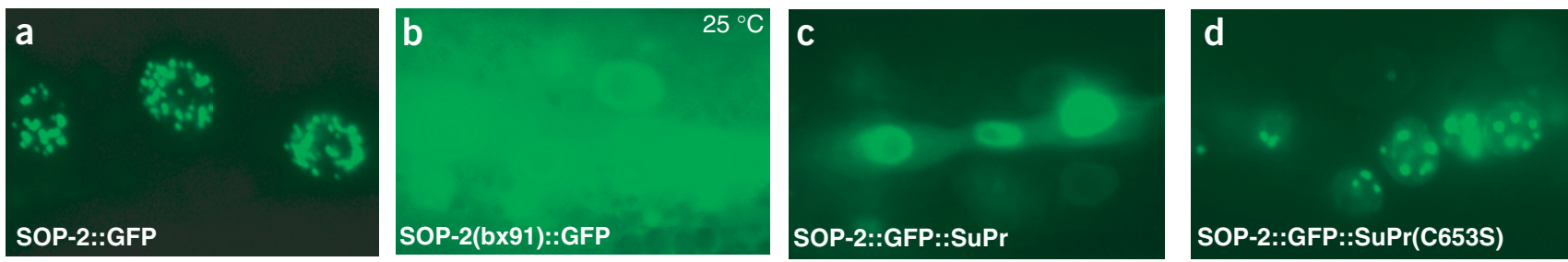

Figure 2 Regulation of SOP-2 nuclear bodies by sumoylation. (a) Expression of SOP-2::GFP in the early adult was localized to nuclear bodies. Expression in hypodermal cells is shown here. (b) Expression of SOP-2(bx91)::GFP in similar cells showed homogeneous nuclear expression. (c) Coexpression of the SUMO protease SuPr with wild-type SOP-2 disrupted the formation of SOP-2 nuclear bodies. (d) Mutating the crucial cysteine to serine (C653S) in the catalytic domain of SuPr restored nuclear bodies.

SOP-2 is functionally analogous to the fly and vertebrate PcG proteins Polyhomeotic $(\mathrm{PH})$ and Sex combs on midleg $(\mathrm{SCM})^{3}$. The limited sequence similarity between SOP-2 and its counterparts is restricted to a protein-protein interaction domain known as the SAM (sterile alpha motif) domain. The SAM domain of SOP-2 mediates its self-association and its localization to nuclear bodies ${ }^{3}$. These distinctive SOP-2 bodies are similar to those formed by the fly and vertebrate $\mathrm{PH}$ orthologs ${ }^{4}$.

To identify proteins that interact with the SOP-2 SAM domain and might contribute to its nuclear localization, we screened a yeast twohybrid C. elegans cDNA library using the $\mathrm{C}$ terminus of SOP-2, which includes this domain. We identified multiple independent clones corresponding to UBC-9, in addition to SOP-2 itself (Fig. 1a). Analysis of deletion constructs showed that UBC-9 binding requires the SAM domain of SOP-2. SOP-2(bx91), a temperature-sensitive loss-of-function mutation (P633S in the SAM domain) reduced binding of SOP-2 to UBC-9 but did not affect dimerization with the wild-type SAM domain (Fig. 1b).

We tested whether binding of UBC-9 leads to the sumoylation of SOP-2 using both in vitro and in vivo systems. An in vitro sumoylation assay using labeled SOP-2 as substrate confirmed sumoylation of wildtype SOP-2 (Fig. 1c). The SUMO conjugation pathway is highly conserved, which allowed us to test SOP-2 sumoylation in vivo, after
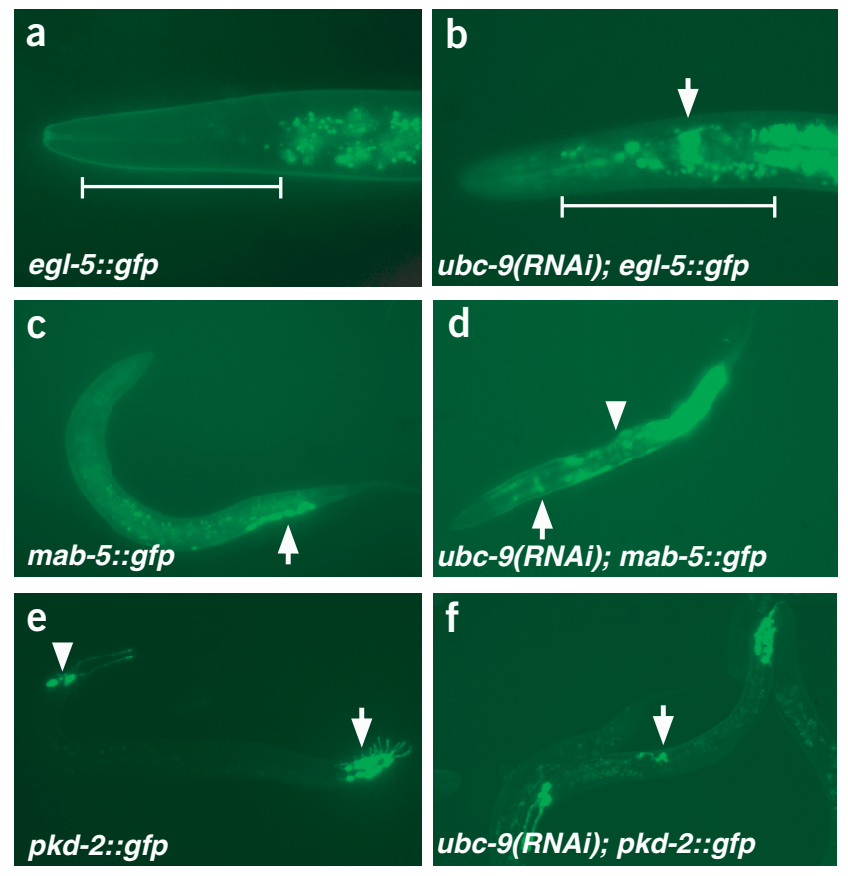

transfecting expression constructs into human U2OS cells. Sumoylation of SOP-2 by the endogenous machinery was evident by immunoprecipitation and western-blot analysis (Fig. 1c). The mutant SOP-2(bx91) protein had markedly less sumoylation both in vitro and in vivo (Fig. 1c), suggesting that the sumoylation of SOP-2 is correlated with its functional properties in vivo.

Whereas wild-type SOP-2 was localized to distinct nuclear bodies, the SOP-2(bx91) mutant protein, which had impaired sumoylation, showed diffuse nuclear expression (Fig. 2a,b). To directly test the requirement for sumoylation in targeting SOP-2 to nuclear bodies, we coexpressed the C. elegans SUMO protease (T10F2.3) along with a SOP2:GFP reporter using a single chimeric construct. The SUMO proteases (SuPr) effectively direct the removal of SUMO moieties from tagged proteins ${ }^{5,6}$. In SOP-2::GFP::SuPr worms, the GFP signal was homogenously distributed throughout the nucleus (Fig. 2c). This distribution requires the enzymatic activity of the protease, as mutating the crucial cysteine in the catalytic domain of SuPr to serine (C653S) restores formation of nuclear bodies (Fig. 2d). Both worms expressing mutant SOP-2(bx91) and those expressing SuPr showed a detectable cytoplasmic GFP signal (Fig. 2b,c), indicating that sumoylation also contributes to the nuclear uptake or nuclear retention of SOP-2. Accumulation of unsumoylated protein in the cytoplasm has also been shown for the fruit fly developmental regulators Dorsal and Bicoid ${ }^{7,8}$.

We analyzed sumoylation in intact worms to test the functional significance of this protein modification in a defined developmental pathway. Inactivation of SOP-2 leads to ectopic expression of Hox genes and homeotic transformations, as shown by the anterior-to-posterior transformation in cell fate adopted by the row of epidermal seam cells in sop2 males $^{3}$. In wild-type adult males, the anterior seam cells V1-V4 produce the cutical structures called alae, whereas the posterior seam cells V5 and V6 give rise to male sensory organs called rays ${ }^{9}$; in sop-2 mutants, anterior seam cells generate rays instead of alae ${ }^{3}$. We observed a similar result after global suppression of the sumoylation pathway (Fig. 3). Injection of worms with double-stranded RNA targeting $u b c-9$

Figure 3 Ectopic expression of Hox genes and homeotic transformation after loss of function of components of the sumoylation pathway. (a) Absence of EGL-5::GFP expression in the head of a wild-type worm (marked with bar). (b) Ectopic expression of EGL-5::GFP in a ubc-9(RNAi) L1 larva. eg/-5 was expressed in many head neurons (arrow) and ventral cord neurons (data not shown). (c) Expression of mab-5 in a wild-type early L1 larva. MAB-5::GFP was confined to a few posterior cells (arrow). (d) Ectopic expression of MAB5::GFP in a ubc-9(RNAi) L1 larva. mab-5 was expressed in the head neurons (arrow) and hypodermal cells (arrowhead). Faint irregular fluorescence in a-d results from gut autofluorescence. (e) Normal expression of PKD-2::GFP in all nine pairs of B-type ray neurons (arrow) and in four head neurons of a wild-type male (arrowhead). (f) Ectopic expression of PKD-2::GFP in the anterior body region in a ubc-9(RNAi) male (arrow). PKD-2::GFP marked both the cell body and the axon of ray neurons. 
Table 1 Defects in loss of function of sumoylation pathway components

\begin{tabular}{|c|c|c|c|}
\hline Genotype & Embryonic lethality & Ectopic expression of eg/- $5^{a}$ & Male sides with ectopic anterior rays \\
\hline Wild-type & $<3 \%(n>500)$ & $0 \%(n>100)$ & $0 \%(n>500)$ \\
\hline sop-2(bx91)b & $<3 \%(n>500)$ & $100 \%(n>100)$ & $99 \%(n=284)$ \\
\hline$u b c-9(R N A i)$ & $74 \%(n=2,330)$ & $45 \%(n=36)$ & $3 \%(n=84)^{c}$ \\
\hline uba-2/aos-1(RNAi) & $75 \%(n=646)$ & $31 \%(n=42)$ & $3 \%(n=54)^{\mathrm{c}}$ \\
\hline sumo(RNAi) & $86 \%(n=708)$ & $53 \%(n=30)$ & ND ${ }^{d}$ \\
\hline
\end{tabular}

${ }^{a}$ Ectopic expression of egl-5::gfp in head neurons of early larvae. ${ }^{b}$ Data from ref. 3. ${ }^{c}$ Homeotic transformations are seen only in worms with partial loss of function. ${ }^{\mathrm{d}}$ None of the sumo(RNAi) worms developed into adults.

(F29B9.6), sumo (K12C11.2) or subunits of the E1 activating enzyme uba-2 (W02A11.4) and aos-1 (C08B6.9; ref. 10) causes expansion of the expression domains of Hox genes, as observed in sop-2 mutants ${ }^{3}$. The Hox gene egl-5, which is normally restricted to the tail region in early larva $e^{9,11}$, became ectopically expressed in head neurons, ventral cord neurons and anterior seam cells in $u b c-9$ (RNAi) worms (Fig. 3a,b and Table 1). Similarly, expression of the Hox gene mab-5, which is normally present only in the posterior region, was substantially increased and also ectopically expressed in head and hypodermal cells (Fig. 3c,d and data not shown). Deregulation of Hox gene expression was also observed in uba-2/aos-1(RNAi) and sumo(RNAi) worms (Table 1). Disruption of the sumoylation pathway also produced homeotic transformations. Complete loss of function of $u b c-9$ and $u b a-2 / a o s-1$ is lethal at the embryonic and early larval stages. We observed homeotic transformations in 3\% of adults that developed from the eggs laid in the first few hours after injection, in which no loss of function or only partial loss of function is expected. We scored these homeotic transformations by visualization of the ray-specific marker $p k d-2: g f p$, indicating the presence of ectopic rays derived from anterior seam cells (Fig. 3e,f and Table 1). No such homeotic transformations were observed in $>2,000$ control worms. We concluded from these experiments that global loss of function of components of the sumoylation pathway produces phenotypes that are similar to those of sop-2 mutant worms.
The role of the SOP-2 SAM domain in mediating its sumoylation and its evolutionary conservation across the phylogenetic spectrum has potential implications for the function of vertebrate PcG proteins. SAM domains can be classified into several subfamilies based on their primary sequence, including the SPM subfamily (present in the $\mathrm{PcG}$ proteins $\mathrm{PH}$, SCM and fly tumor suppressor L(3)MBT) and the SAM/PNT subfamily (present in the vertebrate transcriptional repressor TEL). Phylogenetic analysis indicates that the SAM domain of SOP-2 belongs to a new nematode-specific SAM domain subfamily, which also includes the SAM domain of $\mathrm{K} 04 \mathrm{C} 1.2$ and is more closely related to the SAM/PNT than to the SPM subfamiliy ${ }^{3}$. Nonetheless, the crystal structure shows that the SAM domains of PH and TEL form identical head-to-tail, left-handed helical polymers ${ }^{12,13}$, and the SAM domain of TEL is also required for its binding to UBC-9 and localization to nuclear bodies ${ }^{14}$. To correlate the functional properties of these SAM domains with their effects on nuclear localization, we undertook a series of domain-swapping experiments.

Replacement of the SAM domain of SOP-2 with that of K04C1.2 resulted in homogenous nuclear distribution of the chimeric protein (Fig. 4a). In contrast, substitution of the SOP-2 SAM domain with that of PcG proteins (including fruit fly $\mathrm{PH}$ and SCM and the mouse $\mathrm{PH}$ homolog Rae28) preserved the precise number and size of subnuclear SOP::GFP bodies (Fig. $\mathbf{4 b}, \mathbf{g}$ ). This functional conservation in nuclear localization was correlated with the ability of the PH SAM domain, but
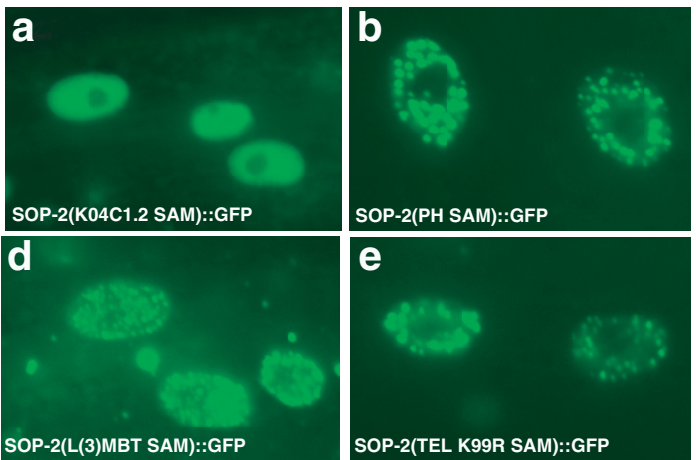
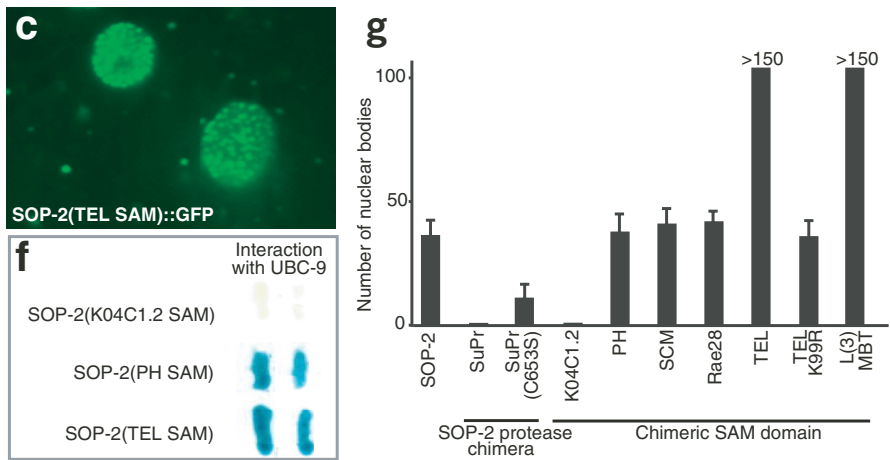

Figure 4 Substitution of the SOP-2 SAM domain with related SAM domains. (a) SOP-2(K04C1.2 SAM)::GFP in early adult showed homogeneous nuclear expression. Expression was excluded from the nucleolus (as for all the reporters described below). (b) Formation of appropriate nuclear bodies by the chimeric SOP-2(PH SAM)::GFP, carrying the SAM domain derived from the PcG PH protein. (c) More and smaller nuclear bodies were produced by the chimeric SOP-2(TEL SAM)::GFP, carrying the SAM domain from TEL, which is more closely related to the SOP-2 SAM domain but has divergent function. (d) Altered number and size of bodies formed by the chimeric SOP-2(L(3)MBT SAM)::GFP. In both SOP-2(TEL SAM)::GFP and SOP-2(L(3)MBT SAM)::GFP, fluorescent bodies were also seen in the cytoplasm of hypodermis cells. (e) Restoration of characteristic SOP-2 nuclear bodies after mutation of the SUMOtargeted Lys99 to arginine in the SAM domain of TEL in SOP-2(TEL SAM)::GFP. (f) Interaction of the SAM domains of K04C1.2, PH and TEL with UBC-9 in a yeast two-hybrid assay. (g) Quantification of nuclear bodies formed in hypodermal cells expressing the chimeric constructs illustrated above, as well as SOP-2 fusion proteins with SuPr and with chimeric SAM domains derived from the PcG genes SCM and Rae28. Data are mean \pm S.e. from analysis of multiple cells expressing SOP-2::GFP $(n=32)$, SOP-2::GFP:SuPr $(n=54)$, SOP-2::GFP::SuPr(C653S) $(n=62)$, SOP-2(K04C1.2 SAM)::GFP $(n=100)$, SOP-2(PH SAM)::GFP $(n=30)$, SOP-2(SCM SAM)::GFP $(n=19)$, SOP-2(Rae28 SAM)::GFP $(n=19)$, SOP-2(TEL SAM)::GFP $(n=6)$, SOP-2(TEL K99R $\operatorname{SAM}):: \operatorname{GFP}(n=18)$ and SOP-2(L(3)MBT SAM)::GFP $(n=6)$ 
Table 2 Rescue of sop-2(bx91) defects by various SAM domain substitution constructs

\begin{tabular}{lrr}
\hline Genotype & Mab (\%) & $n$ \\
\hline Wild-type & 0 & $>2,000$ \\
sop-2(bx91) & 100 & 121 \\
sop-2(bx91); sop-2::gfp & 2 & 134 \\
sop-2(bx91); sop-2(KO4C1.2 SAM)::gfp & 100 & 40 \\
sop-2(bx91); sop-2(PH SAM)::gfp & 8 & 85 \\
sop-2(bx91); sop-2(TEL SAM)::gfp & 96 & 187 \\
sop-2(bx91); sop-2(TEL K99R SAM)::gfp & 3 & 76 \\
\hline
\end{tabular}

Rescue of homeotic transformations by SAM domain chimeras. Growth of sop-2(bx91) mutants at $25^{\circ} \mathrm{C}$ caused a severe Mab (male abnormal) phenotype, due in part to homeotic transformation of rays derived from V 5 and $\mathrm{V} 6$. sop-2(bx91) worms were grown at $20^{\circ} \mathrm{C}$ and shifted to $25^{\circ} \mathrm{C}$ at L1 stage, whereas rescued worms were viable at $25^{\circ} \mathrm{C}$.

not of the K04C1.2 SAM domain, to interact with UBC-9 in the yeast two-hybrid assay (Fig. 4f). The SAM domains of TEL and L(3)MBT, which also bind UBC-9, produced a distinct subnuclear localization pattern, reflected by more and smaller nuclear bodies (Fig. $\mathbf{4 c , d , f , g ) . ~}$ Thus, intrinsic properties of the SAM domain may regulate the characteristics of nuclear body formation. The preferential sumoylation site in TEL, Lys99, is located within the SAM domain ${ }^{14}$, whereas the SAM domains of SOP-2 and PcG proteins lack the corresponding lysine residue and the sumoylated sites in SOP-2 seem to be outside the SAM domain (data not shown). To determine whether the distinct nuclear bodies formed in SOP-2(TEL SAM)::GFP were due to this sumoylation site, we mutated the TEL Lys99 to arginine within the chimeric construct. This construct resulted in the formation of nuclear bodies that were indistinguishable from those of SOP2 itself (Fig. 4e,g). Thus, sumoylation in the oligomerization domain of TEL is responsible for the distinct characteristics of nuclear bodies, possibly through spatial hindrance affecting protein complexes.

To test the functional consequences of distinct nuclear body localization associated with different SAM domains, we injected the chimeric constructs into sop-2(bx91) worms. The developmental defects in sop-2(bx91) males were rescued by expression of SOP-2(PH SAM)::GFP (Table 2). As expected, the closely related but mislocalized SOP-2(K04C1.2 SAM)::GFP did not rescue the defects in the males. SOP-2(TEL SAM)::GFP was also nonfunctional, but the mutation removing the Lys99 sumoylation site in SOP-2(TEL K99R SAM)::GFP almost completely rescued the sop-2 mutant phenotype (Table 2). We conclude from these experiments that correct configuration of nuclear bodies mediated by the SAM domain is essential for the physiological function of SOP-2 and seems to be a property restricted to the SAM domains of PcG family members.

In summary, our observations use both the sequence divergence and the functional conservation between the PcG families in C. elegans and other organisms to define essential features for their physiological role in repressing Hox gene expression. The SAM domain of PcG proteins mediates the interaction with UBC-9, leading to sumoylation, and directs the formation of subnuclear bodies whose characteristics are correlated with physiological function. Sumoylation itself has a variety of effects on its targets in cultured cells, including regulating the subnuclear localization of PML, HIPK2 and TEL ${ }^{14-16}$ and modulating the transactivational activity of Sp3 and LEF1 (refs. 6,17). Moreover, our domain-swapping experiments indicate that distinct nuclear bodies are associated with SAM domains of diverse function. Thus, a subset of SAM domains links sumoylation and formation of nuclear bodies, and different sumoylation signals in the context of these SAM domains seem to target distinct nuclear structures.
Our observations also have implications for understanding the physiological role of sumoylation itself. Various $u b c-9$ mutants have been isolated in the fruit fly, where they have pleiotropic defects, such as suppression of meiotic nondisjunction in lesswright $(l w r)$ mutants and loss of anterior body structure in semushi mutants 8,18 . Our observation that global suppression of sumoylation in C. elegans can mimic the loss of SOP-2 function points to the essential role of sumoylation in Hox gene regulation.

\section{METHODS}

Strains. Unless otherwise stated, we cultured nematodes at $20{ }^{\circ} \mathrm{C}$ and carried out genetic analysis according to standard procedures ${ }^{19}$. Most of the strains we used carry the him-5(e1490) mutation, which produces a high frequency of male self-progeny. We used the following strains: LGII, sop-2(bx91), muIs16(mab-5::gfp, dpy-20(+)); LGIII, pha-1(e2123ts); LGV, bxIs14(pkd-2:gfp, pha-1(+)); LGX, bxIs13(egl-5::gfp, lin-15(+)); extrachromosomal array, bxEx99 (sop-2::gfp (EM\#309), pha-1(+)).

Yeast two-hybrid analysis. We carried out yeast two-hybrid experiments using the ProQuest two-hybrid system (Clontech) as recommended by the manufacturer. We cloned the C-terminal domain of SOP-2 (amino acids 541-735) into vector pPC97 and used it to screen a library of C. elegans cDNAs in the pPC86 vector. We replaced the SAM domain of SOP-2 (amino acids 632-712) in pPC97 with the SAM domains of K04C1.2, PH and TEL to test their interaction with UBC-9 in pPC86. We assessed interactions by scoring blue color on plates of medium containing 5 -bromo-3-indoyl- $\beta$-D-galactopyranoside (X-gal) and confirmed interactions by growth on plates of medium containing 3 -aminotriazole, $\mathrm{Ura}^{-}$and 5-fluoroorotic acid

In vitro sumoylation assay. We transcribed and translated $\mathrm{cDNAs}$ encoding full-length SOP-2 proteins in rabbit reticulocyte lysate in the presence of $\left[{ }^{35} \mathrm{~S}\right]$ methionine according to the manufacturer's instructions (Promega). We purchased the sumoylation kit from LAE Biotech International. We carried out the $20-\mu \mathrm{l}$ sumoylation reactions in a buffer ( $2 \mathrm{mM}$ HEPES, pH $7.5 ; 0.5 \mathrm{mM} \mathrm{MgCl}$ and $0.2 \mathrm{mM}$ ATP). Reactions contained $5 \mu \mathrm{l}$ of the translation product, $375 \mathrm{pg}$ of AOS-1/UBA-2, 25 pg of UBC- 9 and 25 ng of SUMO. After $30 \mathrm{~min}$ of incubation at $30^{\circ} \mathrm{C}$, the reactions were stopped by the addition of an equal volume of SDS sample buffer.

Transfection and immunoprecipitation. We carried out transfection using $6 \mu \mathrm{g}$ of plasmid DNA per dish and Fugene 6 transfection reagent (Roche) and analyzed expression of transfected genes $24 \mathrm{~h}$ after transfection. We washed cells with phosphate-buffered saline and disrupted them with cold lysis buffer (150 mM $\mathrm{NaCl} ; 1 \%$ Nonidet-P40; 50 mM Tris, pH 8.0; 1 mM EDTA; 20 mM N-ethylmaleimide; and $1 \times$ protease inhibitor cocktail, Complete EDTA-free, Roche). We precleared lysates with protein A agarose beads for $2 \mathrm{~h}$ at $4{ }^{\circ} \mathrm{C}$. We added $1 \mu \mathrm{g}$ of antibody to SUMO (D-11, Santa Cruz) to the precleared lysates for $2 \mathrm{~h}$ at $4{ }^{\circ} \mathrm{C}$ and then added fresh protein $\mathrm{A}$ agarose beads and incubated lysates at $4{ }^{\circ} \mathrm{C}$ overnight. We washed the beads three times and eluted bound proteins with $2 \times$ SDS sample buffer and loaded them onto 10\% SDS-PAGE gels. For western-blot analysis, we used antibody to hemagglutinin epitope 16B12 (Covance).

GFP reporters and transgenic line construction. We created mutant forms of SOP-2::GFP by PCR-based mutagenesis. We replaced the SAM domain of the SOP-2::GFP reporter (amino acids 632-712 of SOP-2) with the SAM domains of K04C1.2 (amino acids 424-500), PH (amino acids 1,498-1,577), SCM (amino acids 792-871), Rae28 (amino acids 930-1,010), TEL (amino acids 45-122) and L(3)MBT (amino acids 1,289-1,367). We injected constructs (10 ng $\left.\mu \mathrm{l}^{-1}\right)$ and pBX-1(pha-1) DNA (100 ng $\mu^{-1}$ ) into pha-1(e2123ts); him5(e1490). We constructed sop-2::gfp::supr by inserting the supr cDNA into the SplI site of EM\#309 to create a translational fusion protein containing SuPr in the $\mathrm{C}$ terminus of SOP-2. Expression of the transgene is mosaic, with expressing cells restricted primarily to the head and male tail region, suggesting that ectopic expression of SuPr may be toxic in other tissues. We introduced the mutation C653S in SuPr into sop-2::gfp::supr by PCR-based mutagenesis. We used at least three independent transgenic lines for each construct in this study. 
RNA interference experiments. We carried out RNA interference experiments as described ${ }^{20}$. The primer sequences used to amplify DNA template for synthesizing RNA are available on request. Single-stranded RNA was transcribed using MEGAscript T3 and T7 kits (Ambion), annealed and injected into wildtype, bxIs 13 and muIs 16 worms. F1 progeny generated in the first $0-48 \mathrm{~h}$ were scored for larval lethality and ectopic expression of Hox gene reporters. In $u b c$ 9(RNAi) or uba-2/aos-1(RNAi) worms, eggs laid between $0-12 \mathrm{~h}$ showed embryonic lethality with some escapers developing into adults, whereas $100 \%$ of eggs laid at $12-48 \mathrm{~h}$ showed embryonic lethality. Escapers probably resulted from incomplete loss of function of the sumoylation pathway, rather than from incomplete penetrance of loss-of-function phenotypes.

Accession numbers. Entrez Protein: K04C1.2, CAA94213; PH, P39769; SCM, AAB57632; Rae28, Q64028; L(3)MBT, AAF56691. GenBank: TEL, NM_001987.

\section{ACKNOWLEDGMENTS}

This work was supported by grants from the US National Institutes of Health (to H.Z., S.v.d.H. and D.A.H.).

\section{COMPETING INTERESTS STATEMENT}

The authors declare that they have no competing financial interests.

Received 20 December 2003; accepted 18 February 2004

Published online at http://www.nature.com/naturegenetics/

1. Seeler, J.S. \& Dejean, A. SUMO: of branched proteins and nuclear bodies. Oncogen 20, 7243-7249 (2001).

2. Wilson, V.G. \& Rangasamy, D. Intracellular targeting of proteins by sumoylation. Exp. Cell Res. 271, 57-65 (2001).

3. Zhang, $H$. et al. Global regulation of Hox gene expression in C. elegans by a SAM domain protein. Dev. Cell 4, 903-915 (2003).

4. Netter, S., Faucheux, M. \& Theodore, L. Developmental dynamics of a polyhomeoticEGFP fusion in vivo. DNA Cell Biol. 20, 483-492 (2001).

5. Best, J.L. et al. SUMO-1 protease-1 regulates gene transcription through PML. Mol.
Cell 10, 843-855 (2002).

6. Ross, S., Best, J.L., Zon, L.I. \& Gill, G. SUMO-1 modification represses Sp3 transcriptional activation and modulates its subnuclear localization. Mol. Cell 10, 831-842 (2002)

7. Bhaskar, V., Valentine, S.A. \& Courey, A.J. A functional interaction between dorsal and components of the Smt3 conjugation machinery. J. Biol. Chem. 275, 4033-4040 (2000).

8. Epps, J.L. \& Tanda, S. The Drosophila semushi mutation blocks nuclear import of bicoid during embryogenesis. Curr. Biol. 8, 1277-1280 (1998).

9. Emmons, S.W. Cell fate determination in Caenorhabditis elegans ray develpment. in Cell Lineage and Fate Determination (ed. S.A. Moody) 139-155 (Academic, San Diego, 1999).

10. Jones, D., Crowe, E. Stevens, T.A. \& Candido, E.P. Functional and phylogenetic analysis of the ubiquitylation system in Caenorhabditis elegans: ubiquitin-conjugating enzymes, ubiquitin-activating enzymes, and ubiquitin-like proteins. Genome Biol. 3(1), research0002.1-0002.15 (2002).

11. Ferreira, H.B., Zhang, Y.H., Zhao, C. \& Emmons, S.W. Patterning of Caenorhabditis elegans posterior structures by the Abdominal-B homolog, egl-5. Dev. Biol. 207, 215-228 (1999).

12. Kim, C.A. et al. Polymerization of the SAM domain of TEL in leukemogenesis and transcriptional repression. EMBO J. 20, 4173-4182 (2001).

13. Kim, C.A., Gingery, M., Pilpa, R.M. \& Bowie, J.U. The SAM domain of polyhomeotic forms a helical polymer. Nat. Struct. Biol. 9, 453-457 (2002).

14. Chakrabarti, S.R., Sood, R., Nandi, S. \& Nucifora, G. Posttranslational modification of TEL and TEL/AML1 by SUMO-1 and cell-cycle-dependent assembly into nuclear bodies. Proc. Natl. Acad. Sci. USA 97, 13281-13285 (2000).

15. Zhong, S., Salomoni, P. \& Pandolfi, P.P. The transcriptional role of PML and the nuclear body. Nat. Cell Biol. 2, E85-E90 (2000).

16. Kim, Y.H., Choi, C.Y. \& Kim, Y. Covalent modification of the homeodomain-interacting protein kinase 2 (HIPK2) by the ubiquitin-like protein SUMO-1. Proc. Natl. Acad. Sci. USA 96, 12350-12355 (1999).

17. Sachdev, S. et al. PIASy, a nuclear matrix-associated SUMO E3 ligase, represses LEF1 activity by sequestration into nuclear bodies. Genes Dev. 15, 3088-3103 (2001).

18. Apionishev, S., Malhotra, D., Raghavachari, S., Tanda, S. \& Rasooly, R.S. The Drosophila UBC9 homologue lesswright mediates the disjunction of homologues in meiosis I. Genes Cells 6, 215-224 (2001).

19. Brenner, S. The genetics of Caenorhabditis elegans. Genetics 77, 71-94 (1974)

20. Fire, A. et al. Potent and specific genetic interference by double-stranded RNA in Caenorhabditis elegans. Nature 391, 806-811 (1998). 